\title{
Manejo de errores en un dominio de conocimiento y la competencia para la resolución de problemas"
}

\author{
Error handling in a domain knowledge and problem - solving competence \\ Manejo de erros num domínio de conhecimento e a con- \\ corrência de resolução de problemas
}

Recibido: mayo de 2012 Aprobado: julio de 2012
Luis Eduardo Otero Sotomayor ${ }^{2 * *}$

\begin{abstract}
Resumen
El manejo de errores es importante para el aprendizaje de conceptos y el desarrollo de la competencia de resolución de problemas. La retroalimentación es relevante en el análisis de errores, ya que apoya procesos de aprendizaje eficientes en los ambientes basados en el uso del computador. Bajo este panorama, el estudio que se condujo es de tipo explicativo, cuasiexperimental, cuya variable independiente es un objeto de aprendizaje caracterizado por el manejo de errores. La variable dependiente es el efecto sobre la comprensión de un dominio de conocimiento y la competencia de resolución de problemas. Se encontró que no hubo diferencia significativa entre un grupo que siguió la clase magistral (grupo B) y otro que fue expuesto al objeto de aprendizaje (grupo A). Para el grupo A los resultados se vieron afectados por el poco interés de leer la retroalimentación y escaso avance en los niveles propuestos, de 4 alcanzaron el 2.
\end{abstract}

Palabras clave: objeto de aprendizaje, dominio de conocimiento, competencia de resolución de problemas

\begin{abstract}
Error handling is important in concepts learning and the development of problem-solving competence. Feedback is relevant in error analysis, in supporting efficient learning processes in computer-based environments. The study conducted is of explanatory, quasi - experimental type, whose independent variable is an object of learning characterized by handling errors. The dependent variable is the effect on the understanding of a domain knowledge and problem-solving competence. It was found that there was no significant difference between a group that followed the lecture (group B) and one that was exposed to the learning object (group A). For group A the results were affected by the lack of interest to read the feedback and lacks of progress in the proposed levels, of 4 levels, 2 were attained.
\end{abstract}

Keywords: learning object, domain knowledge, problem-solving competition

1 *Esta publicación tiene su origen en una investigación anterior referente al manejo de errores, efectuada en un colegio de Bogotá

$2 \quad{ }^{* * *}$ Docente de UNITEC. Proyecto de investigación pedagógica en el área de las matemáticas. Correo electrónico: investigador4@ unitec.edu.co 


\section{Resumo}

Manipulação de erro é importante na aprendizagem de conceitos e desenvolvimento de solução de problemas de concorrência. O feedback é importante na análise de erros, como suportes eficientes os processos de aprendizagem em ambientes baseados em computador. O estudo realizado é do tipo explicativa, quase - experimental, cuja variável independente é um objeto de aprendizagem caracterizado pela manipulação de erros. A variável dependente é o efeito sobre a compreensão de um domínio de conhecimento e resolução de problemas de concorrência. Descobrimos que não houve diferença significativa entre um grupo que se seguiu à palestra (grupo B) e outra que foi exposto ao objecto de aprendizagem (grupo A). Para o Grupo A os resultados foram afetados pela falta de interesse de ler os comentários e pouco progresso nos níveis propostos de 4 atingiu 2.

Palavras-chave: objeto de aprendizagem, o conhecimento de domínio, a competição de resolução de problemas

\section{Introducción}

\section{Formulación del problema de investigación}

De acuerdo con Mory, un error ofrece la oportunidad invaluable de clarificar los malos entendidos en el aprendizaje; también juega un papel importante en los procesos de retroalimentación. Según Noonan (1984), citado por Mory (2004), son más útiles los procedimientos sofisticados relacionados con el análisis de errores que la tradicional retroalimentación de la respuesta correcta. Los ambientes de aprendizaje -basados en el computador-facilitan el almacenamiento y análisis de los diferentes tipos de errores, brindando una adecuada retroalimentación. Según Narciss (2001), esta última se considera como fuente de información necesaria, especialmente cuando el estudiante encuentra obstáculos o procede incorrectamente.

Skinner (1958), a través de su instrucción programada, utilizaba la retroalimentación para modelar conductas, reforzando las respuestas deseadas e ignorando o considerando como una aversión los errores en la instrucción. Para lograrlo llevaba al aprendiz paso a paso, a través del material, dándole la retroalimentación como refuerzo y motivación. Posteriormente, muchos investigadores comenzaron a dudar de los beneficios de la retroalimentación, desde el punto de vista del refuerzo (Mory,
2000). Así mismo, cambios en la tendencia hacia la psicología cognitiva condujeron a los investigadores a concentrarse en cómo la retroalimentación influenciaba los procesos cognitivos y meta cognitivos en el aprendiz (Kulhavy, 1977, citado por Mory, 2000).

Desde el punto de vista del procesamiento de la información, el aprendiz participa en el sistema mediante la corrección de sus errores, en otras palabras, a través de una función correctiva. Según Kulhavy y Stock (1989), una retroalimentación efectiva le brinda al estudiante dos tipos de información: de verificación y de elaboración. La segunda conduce al estudiante a obtener la respuesta correcta. Así, puede ser que le facilite un marco de información relevante de donde puede obtener la respuesta correcta (elaboración informacional); que se le conduzca a la respuesta correcta sin tener en cuenta las incorrectas (elaboración especifica al tópico); o que se explique, en caso de error, por qué una respuesta es incorrecta, dando además información de la respuesta correcta (elaboración específica a la respuesta).

Justamente en la elaboración específica a la respuesta es en donde se concentra la presente investigación. El manejo de errores que efectúa el objeto de aprendizaje a utilizar, no solamente explica por qué la respuesta no es correcta (en términos de la respuesta correcta, en caso de error), sino que 
explica el concepto con el que se ha confundido la solución correcta. Brindado así una información que no solamente apunta a enseñar sobre la pregunta que se está resolviendo, sino al tema que se quiere aprender.

Para el estudio se utilizará un objeto aprendizaje que de acuerdo con Wiley (2000), citado por María Elena Chan (2001), es "cualquier recurso digital que puede ser re usado como soporte para el aprendizaje". En Colombia, el Ministerio de Educación Nacional (en adelante MEN), en los últimos años, ha desarrollado lineamientos de política educativa para fomentar el diseño y uso de tales objetos (Universidad Javeriana, 2009). Del mismo modo, en el ámbito internacional se puede encontrar diversa literatura que se centra en los componentes de estos objetos de aprendizaje, incluso existen comités de estandarización de diseño (Calhoun Beverly y Wooten, 2003). Wagner, citado por Mory (2004) afirma que es un cambio de la perspectiva conductista a la cognoscitiva; y desde la perspectiva objetivista a la constructivista (Wagner, 2002).

En este trabajo se desea, a través de la utilización de un objeto de aprendizaje, establecer el efecto del manejo de errores en la comprensión de la teoría y desarrollo de la competencia de resolución de problemas en estudiantes. Con tal fin, se plantea la siguiente pregunta: ¿Cuál es el efecto del manejo de errores en la comprensión de un dominio de conocimiento y en la competencia de resolución de problemas en estudiantes de primer semestre de ingeniería? De donde se obtienen las siguientes sub preguntas:

- ¿Cuál es el nivel de comprensión del dominio de conocimiento, alcanzado por un grupo de estudiantes expuesto a la enseñanza tradicional?

- ¿Cuál es el nivel de competencia en resolución de problemas, alcanzado por un grupo de estudiantes expuesto a un objeto de aprendizaje?

- ¿Cuál es el nivel de competencia en resolución de problemas, alcanzado por un grupo de estudiantes expuesto a la enseñanza tradicional?
- ¿Cuál es el nivel de comprensión del dominio de conocimiento, alcanzado por un grupo de estudiantes expuesto a un objeto de aprendizaje?

- ¿Existen diferencias significativas en el desempeño de dos grupos de estudiantes: uno expuesto a la enseñanza tradicional y otro a un objeto de aprendizaje?

\section{Objetivos}

\section{Objetivo general}

Determinar el efecto del manejo de errores en la comprensión de un dominio de conocimiento y en la competencia de resolución de problemas en estudiantes de primer semestre de ingeniería.

\section{Objetivos específicos}

- Determinar el nivel de comprensión del dominio de conocimiento, alcanzado por un grupo de estudiantes expuesto a la enseñanza tradicional.

- Determinar el nivel de competencia en resolución de problemas, alcanzado por un grupo de estudiantes expuesto a un objeto de aprendizaje

- Determinar el nivel de competencia en resolución de problemas, alcanzado por un grupo de estudiantes expuesto a la enseñanza tradicional

- Determinar el nivel de comprensión del dominio de conocimiento, alcanzado por un grupo de estudiantes expuesto a un objeto de aprendizaje

- Establecer si existen diferencias significativas en el desempeño de dos grupos de estudiantes: uno expuesto a la enseñanza tradicional y otro a un objeto de aprendizaje.

\section{Justificación}

La justificación se puede mirar desde dos puntos de vista: el uso del computador en la educación y desde el uso del manejo de errores como medio 
para mejorar la competencia de resolución de problemas en los estudiantes.

Desde el primer aspecto, los avances en la tecnología han traído consigo cambios en el paradigma de cómo se aprende. Por ejemplo, la necesidad de adaptar el material instruccional utilizado en clase y de crear una instrucción de calidad de bajo costo basado en la web, ha dado lugar a los objetos de aprendizaje que se pueden usar en múltiples contextos. De acuerdo con Bannan y Murphy (2000), citados por Chan (2001), se habla mucho de los atributos tecnológicos de los objetos, de los estándares para los metadatos y especificaciones referentes a la granularidad e interoperabilidad, pero no se ha analizado la necesidad de conocer las implicaciones de usar estos objetos en la educación.

En este orden de ideas, las definiciones de objetos de aprendizaje pueden ser muy variadas, aunque, siguiendo a Chan (2001), lo que da identidad de aprendizaje no es la información temática, sino la actividad sobre esa información. Esto se logra si su diseño se base en hallazgos de la sicología del aprendizaje o la ciencia cognitiva, enriquecido con los principios de creación de los tutores inteligentes.

Por otro lado, la tendencia general es modificar el proceso de enseñanza para que se convierta en un proceso de aprendizaje. Así, por ejemplo, las nuevas prácticas pedagógicas están centradas en el estudiante. Pero además hay múltiples razones que justifican el uso de los objetos de aprendizaje, entre ellas se pueden traer a colación las mencionadas por María Elena Chan (2001):

- El cambio paulatino del paradigma educativo, centrado en la enseñanza, ha orientado el aprendizaje.

- La necesidad de diversificación y flexibilización de la oferta educativa con el consecuente impulso de las modalidades educativas abierta y a distancia.

- El reconocimiento de la necesidad de flexibilizar el currículum en las instituciones educativas de nivel superior.
- La presión para la internacionalización de los programas educativos, que obligan a mirar los criterios y estándares de calidad generados por organismos certificadores en torno a competencias de los egresados.

- La mayor aceptación de la tecnología como factor de innovación educativa por parte de los docentes y los estudiantes.

- La presión para actualizar continuamente los contenidos educativos, con forme al acelerado avance tecnológico.

- Las políticas orientadas hacia la optimización de recursos públicos destinados a educación, a través de la generación de proyectos interinstitucionales.

Sumado a lo anterior, un aspecto clave del uso de los objetos de aprendizaje es la retroalimentación y, dentro de esta, el manejo de errores. Así, uno de los efectos más importantes de la retroalimentación es identificar los errores y conceptos equivocados.

\section{Marco referencial}

De acuerdo con Mory (2004), la perspectiva actual considera el error como una oportunidad importante para clarificar los malos entendidos en el aprendizaje, convirtiéndolo en un factor importante para la retroalimentación. La creencia que la función de la retroalimentación consiste en corregir errores, hace que sea una función crítica en el proceso correctivo. Phye y Bender (1989), citados por Mory (2004), dividen los eventos que pueden ocurrir cuando se usa retroalimentación informativa en diseños de retroalimentación (pre y postest) en tres funciones: función confirmatoria, función correctiva y no funcional. El primero de estos, confirma una respuesta correcta en el pretest (una combinación de correcto - correcto). El segundo, cuando la función es correctiva, corrige errores en el pretest (una secuencia de equivocado - correcto). El tercero ocurre cuando no cumple ninguna función, en cuyo caso aparecen errores en postest.

Así mismo, cuando la retroalimentación no es funcional pueden ocurrir tres tipos de errores. El 
primero lo constituye el mismo error y es perseverante en su naturaleza. Esto quiere decir, que el mismo error que se cometió en el pretest se vuelve a repetir en el postest sin que la retroalimentación produzca algún efecto. El segundo tipo de error ocurre cuando no se tiene en cuenta algún elemento en el pretest y el error es diferente a través de los ensayos. El último (tercero) es un tipo de error en donde la respuesta fue buena en pretest pero cambio a errónea en el postest; tal vez, porque en el pretest se adivinó la respuesta.

A partir de lo anterior, podemos decir que las combinaciones posibles de respuestas pre y postest son: 1) correcto - correcto, 2) error - correcto, 3) error - el mismo error, 4) error diferente error, 5) correcto - nuevo equivocado.

En lo que concierne al aprendizaje de conceptos, los errores de clasificación del concepto se presentan como errores de "generalización por encima", "generalización por debajo" y "concepciones equivocadas" (Mory, 2004). Por ejemplo, cuando un estudiante está aprendiendo a clasificar un miembro de un concepto, debe hacer discriminación entre ejemplo y no ejemplos. Igualmente, existen unos "no ejemplos" que son difíciles de discriminar como ejemplo del concepto, a estos se les llama "no ejemplos cercanos"; otros, en cambio, pueden ser fáciles de discriminar y se les conoce como "no ejemplos lejanos" (Dempsey, 1988, citado por Mory, 2004). Cuando un estudiante comete el error de "generalización por encima", al aceptar "no ejemplos", se dice que tiene una discriminación fina. Esta ocurre cuando "no ejemplos cercanos" se clasifican como "ejemplo del concepto". Si el estudiante clasifica "no ejemplos lejanos" como "ejemplos del concepto", puede estar "generalizando por debajo", dando como resultado una discriminación gruesa del concepto. Dempsey (1988), citado por Mory (2004), desarrolló un estudio para demostrar que ocurren más errores de discriminación fina que gruesa.

De acuerdo con Mory, en la corrección de errores la retroalimentación tiene los efectos más promisorios. Bajo esta perspectiva, los investigadores deben examinar maneras para manipular la retroalimentación y maximizar así los eventos de aprendizaje. Del mismo modo, los procedimientos que tiene que ver con el análisis de errores comunes pueden ser más útiles que la tradicional retroalimentación de respuestas correctas. La información de retroalimentación adaptativa se puede facilitar para trabajar en ambientes basados en el uso del computador, en donde este instrumento puede analizar los tipos de errores que se cometen y, a partir de allí, brindar la retroalimentación pertinente.

\section{Retroalimentación}

Como se hemos podido observar, la retroalimentación desempeña un papel importante en el análisis de errores. Se sugiere, sin embargo, consultar a Otero (2009), Mory (2004) y Narciss (2004) para profundizar más acerca de esta temática.

De acuerdo con Narciss (2004), la retroalimentación es un factor importante para el apoyo de procesos de aprendizaje eficientes, en especial, aquellos que se fundamentan en el uso del computador como instrumento de enseñanza. Bajo esta perspectiva, no solamente es plausible la utilización de tipos de retroalimentación como $\mathrm{KR}$ (que informa si la respuesta es correcta), KCR (que provee la respuesta correcta) o AUC (que presenta la misma pregunta hasta que se responda correctamente), sino también, la retroalimentación de tutorías informativas (ITF). Esta última se refiere al tipo que provee información estratégica útil para completar las tareas, sin ofrecer de inmediato la solución correcta. Sus componentes pueden ser señales para recuperar hechos, analogías, ejemplos trabajados, sugerencias sobre posibles fuentes de información, fuente del error, estrategias metacognitivas y preguntas socráticas.

\section{La competencia de resolución de problemas y el dominio disciplinar}

Jonassen (2000) afirma que la competencia en resolución de problemas es específica al dominio y depende del nivel de conocimiento de quienes resuelvan los problemas. En este orden de ideas, lo que conozca una persona sobre el dominio es importante para 
entender el problema y generar soluciones. Para esto, el conocimiento del dominio debe estar bien integrado para apoyar la resolución. Además, la integración del conocimiento del dominio se conoce como "conocimiento estructural", lo cual se refiere a la manera como los conceptos se interrelacionan en un dominio. Del mismo modo, la organización de las relaciones entre los conceptos en la memoria a largo plazo se denomina "estructura cognitiva" (Shavelson, 1972, citado por Jonassen, 2000).

Pos su parte, Rohrer y Suen (1999), señalan que el conocimiento estructural es un tipo de conocimiento que facilita la traducción del conocimiento de dominio en conocimiento procedimental, en otras palabras, es como una persona representa su entendimiento de una disciplina. Este consta de tres componentes: 1) conceptos del dominio de conocimiento, 2) la presencia de relación entre los conceptos y 3 ) la fortaleza de esas relaciones.

Sumado a lo anterior, la presencia y naturaleza entre los conceptos se conoce como "estructura del conocimiento". Así, cuando los conceptos están interrelacionados, se pueden recuperar más fácilmente de la memoria; y una manera de enlazar los conceptos es a través de las relaciones estructurales. Es decir, el significado de un concepto se deriva del significado de sus partes y estos, a su vez, se enlazan más, debido a la existencia de relaciones más altas.

\section{Materiales y métodos}

Como se mencionó al inicio de este artículo, la presente investigación es de tipo explicativo cuasiexperimental, ya que, mediante el planteamiento de una relación causal entre las variables, pretende determinar los posibles efectos de un objeto de aprendizaje sobre el aprendizaje de la teoría de la línea recta y su aplicación en problemas. Dado que el procedimiento de aleatorización se empleó en gran parte del proceso investigativo, salvo en la selección de los participantes del estudio (por la limitación de asignar los participantes en forma aleatoria a los grupos experimentales), la investigación se encuentra en el nivel cuasi experimental.

\section{Diseño}

Teniendo en cuenta que la investigación contiene una sola variable independiente objeto de aprendizaje, el estudio se clasifica dentro de los diseños cuasiexperimentales variables univalentes. Se empleará un diseño "pretest - test - postest" con dos grupos intactos: un grupo experimental (al que se le aplica el objeto de aprendizaje) y otro de control (al cual no se le aplica).

\section{Participantes}

Estudiantes de educación media (grados $8^{\circ}, 9^{\circ} \mathrm{y}$ $10^{\circ}$ ) del colegio Psicopedagógico Angeli, a quienes se les expuso el tema de línea recta y su aplicación para la solución de problemas. Se tomaron dos cursos como grupos intactos: uno funcionó como grupo experimental (aleatoriamente se le expuso el objeto de aprendizaje) y otro (al cual no se le aplicó) recibió clases de la manera tradicional.

\section{Instrumentos}

Se suministró a los estudiantes participantes una prueba de entrada para evaluar los conocimientos sobre la línea recta, esto permitió establecer las diferencias de conocimiento previo. Luego se aplicó el tutor del objeto de aprendizaje y después se hizo una evaluación sobre los aspectos teóricos. Posteriormente, se sometió a los estudiantes a la resolución de problemas para, mediante una prueba final, verificar el efecto del objeto aplicado.

\section{Objeto de aprendizaje}

Está constituido por tres partes: "presentación", "dominio de conocimiento" y "resolución de problemas". En la "presentación" se hace una descripción del software y de la sintaxis que se debe aplicar para su manejo. El "dominio de conocimiento" se compone de un tutor y un documento resumen sobre la línea recta y las partes que la integran. Al terminar el estudio del tutor se facilita una evaluación para verificar el grado de aprendizaje de la teoría. 
La tercera parte o "resolución de problemas" está divida en tres secciones: comprensión, definición de variables y modelación matemática. Cada una de estas partes tiene cuatro niveles de dificultad que se describen a continuación.

En el primer nivel, en el apartado de comprensión, se pretende que el estudiante reconozca -dentro del contexto del problema- las variables dependientes e independientes, para que luego sea capaz de comprender que el objetivo del problema está ligado a la variable dependiente, la cual es una consecuencia del comportamiento de la variable independiente. En el mismo nivel, en el apartado de definición de variables, se hacen corresponder las definiciones de las variables con las descripciones que están dadas en el problema. Finalmente, para este nivel, la modelación matemática consiste en reemplazar el valor de la variable independiente en una ecuación dada en el problema, para conocer el valor de la variable dependiente.

En el segundo nivel, el apartado de comprensión tiene como fin ayudar al estudiante a reconocer la pendiente y el punto de corte en problemas cuyas variables tienen una relación lineal. En la definición de variables se deben definir las variables y escoger, dentro de una lista, la descripción que le corresponda a cada una. En la modelación matemática se debe dar la línea recta, reemplazando en la ecuación general la pendiente y el punto de corte, previamente reconocidos dentro del problema.

En el tercer nivel, en el apartado de comprensión se desea que el estudiante ubique, dentro del contexto del problema, un punto y la pendiente. Con estos datos, posteriormente, hallará el punto de corte y la ecuación de la línea recta que representa la situación descrita en el problema. Finalmente, en el cuarto nivel, se pide identificar la parte de la comprensión, a partir de dos puntos dados. Luego, en la parte de modelación matemática, se hallará la pendiente, el punto de corte y la ecuación.

La parte de comprensión intenta lograr su objetivo a través de preguntas con opciones múltiples. Cada opción representa un concepto, de tal manera que cuando el estudiante escoge la respuesta equivocada se le brinda retroalimentación informativa para que aprenda sobre el concepto que describe la opción y su diferencia con la respuesta correcta. En la definición de variables y la modelación matemática, las preguntas son abiertas, pero también se maneja el error, aclarando las confusiones y dando sugerencias que conduzcan a encontrar la solución correcta.

\section{Variables}

La variable independiente la constituye el objeto de aprendizaje, que se caracteriza por el manejo de errores, la retroalimentación de retorno diferencial y su interactividad; esta última se refiere a los elementos básicos que establecen la diferencia con el tipo de enseñanza tradicional.

A partir de aquí se definen las siguientes variables adicionales que ayudarán a establecer el efecto del objeto de aprendizaje sobre la comprensión de la teoría de la línea recta y la resolución de problemas sobre el mismo tema.

Manejo de errores. Como variable denota el efecto que tiene la corrección sobre el desempeño del estudiante y mide cuántas veces se corrige un error similar hasta que se asimile el principio correcto. El error se aprovecha para que el estudiante obtenga un aprendizaje y logre despejar las confusiones sobre los diferentes conceptos.

Dominio de conocimiento. Se mide con el resultado de la evaluación sobre dominio de conocimiento.

Competencia de resolución de problemas. Se mide a través del desempeño mostrado en la resolución de problemas, obtenido en la prueba escrita que se hizo al final del experimento.

\section{Procedimiento}

Los grupos se constituirán por los estudiantes que conforman dos cursos. Aleatoriamente, un curso será el grupo A y otro el grupo B. Estos resultados serán analizados con el paquete estadístico SPPS 
19.0 para Windows. Del mismo modo, los contrastes de hipótesis se llevarán a cabo tomando como base la distribución $t$ de student (que se acerca a la distribución $\mathrm{z}$ cuando la muestra es grande) con un nivel de confianza del $95 \%$ y un nivel de significación del 0,05 para contrastes de dos colas mediante diferencia de medias. Para comprobar el desempeño entre los momentos de entrada y salida del experimento se usará la prueba de rango con signo de Wilcoxon, la cual es una alternativa a la prueba paramétrica apareada. También se realizará la prueba apareada con $t$ de Student a modo de comparación. En este caso, cada unidad experimental genera dos observaciones, sobre las cuales se obtendrá una perspectiva acerca de sus diferencias (Anderson, Sweeney, Williams, 2005).

Para controlar las diferencias de conocimiento previo, se administrará un pre test con 9 puntos, los cuales contendrán aspectos teóricos y problemas para su solución. Luego, se les pedirá a los estudiantes estudiar un tutor y documento sobre la línea recta que forman parte del objeto de aprendizaje durante 3 sesiones, de 2 horas. Seguido, resolverán una evaluación de 9 puntos de selección múltiple, con múltiplos intentos, sobre los fundamentos de la línea recta, contenidos en el objeto de aprendizaje como actividad de aprendizaje y luego se les aplica una evaluación sobre los aspectos teóricos. Para que finalmente, resuelvan los problemas que los prepara para presentar el post test.

\section{Resultados y discusión}

\section{Población, muestra y procedimientos experimentales}

Inicialmente había una muestra con 34 estudiantes del colegio Psicopedagógico Angeli, pertenecientes a los grupos $8^{\circ}, 9^{\circ}$ y $10^{\circ}$. Estos se distribuyeron aleatoriamente en dos grupos: el grupo A trabajó con el objeto de aprendizaje, mientras que al grupo B se impartió la clase tradicional. Sin embargo, el día del postest asistieron 16 individuos; la experimentación se realizó durante 6 días, cada grupo vino 3 tres días, tres horas diarias, incluido el tiempo de evaluación, que se realizó el último día. A cada participante se le entregó la prueba, previa terminación de su mediación con el objeto (grupo A) o la terminación de la clase (para el caso del grupo B).

Bajo esta perspectiva, los estudiantes del grupo A enfrentaron un objeto de aprendizaje que les ofrecía la posibilidad de adquirir conocimientos y fortalecer la competencia de resolución de problemas referentes a la línea recta. Por otro parte, los estudiantes del grupo B se sometieron a clase magistral, utilizada durante mucho tiempo para la formación de profesionales. Luego se hizo una comparación con este grupo, con el fin de crear alternativas de formación basadas en las características tecnológicas que ofrece el mundo contemporáneo. El experimento terminó con una prueba en donde cada pregunta tenía como propósito inducir en el estudiante la importancia de construir esquemas, necesarios para la resolución de problemas.

\section{Datos y resultados de la experimentación}

El contraste de hipótesis se llevó a cabo tomando como base la distribución $t$ student con un nivel de confianza del $95 \%$ y un nivel de significación de 0,05 para contrastes de dos colas mediante diferencia de medias. Para contrastar la hipótesis de la no existencia de diferencia significativa entre los grupos se utilizó el paquete estadístico SPPS 19.0 para Windows. En este se pueden presentar dos casos: cuando se asume la igualdad de varianzas y cuando hay diferencias entre ellas. La regla de decisión depende del valor de Sig. tal y como se describe a continuación:

- Si Sig. > 0,05; no hay diferencia significativa entre las varianzas

- Sig. $<0,05$; hay diferencia significativa

Luego, según el caso se lee el valor de Sig. para dos colas y se aplica la misma regla:

- Si Sig. > 0,05; no hay diferencia significativa entre las medias de las muestras

- Sig. < 0,05; hay diferencia significativa

En este caso, se determina si hay diferencia significativa entre las medias. La columna Sig. representa la probabilidad de que la diferencia en el 
estadístico observado se deba al azar. El estadístico es significativo cuando el valor de Sig. es menor que la probabilidad escogida para el error tipo I, alfa. Comúnmente, alfa se escoge de 0,05.

Comparación prueba de entrada entre grupo A y B. De acuerdo con los resultados estadísticos (Sig. 0,866), no se encontró diferencia significativa en el conocimiento existente en los dos grupos de estudiantes, antes de comenzar la experimentación.

Comparación prueba de entrada y salida. Hubo diferencia significativa entre las notas del examen de entrada y salida, a favor del desempeño mostrado en el examen de salida (Sig. 0,00). Lo anterior quiere decir que los estudiantes en general, independientemente del grupo, aprovecharon las clases sobre la línea recta.

Comparación prueba de entrada y salida, grupo A. Tomando cada grupo por separado, el grupo A mostró diferencia significativa entre los dos momentos de evaluación, a favor del desempeño mostrado en el examen de salida con Sig. de 0,022. El objeto de aprendizaje facilitó esta diferencia.

Comparación prueba de entrada y salida. El grupo B, que tomó las clases magistrales, tuvo un desempeño significativo en la prueba de salida, comparada con la prueba de entrada (Sig. 0,017).

Comparación prueba de salida entres los grupos A y B. No hubo diferencia significativa entre los grupos. Ante este resultado se debe aclarar que el grupo A solo llegó al nivel 2, comparado con el grupo $\mathrm{B}$ que cubrió todos los niveles programados. Cuando un profesor regenta la clase impone el ritmo de ésta. En un objeto de aprendizaje, cada participante va a su paso.

\section{Conclusiones}

\section{Efecto del objeto de aprendizaje línea sobre el conocimiento del dominio y la competencia para la resolución de problemas}

De acuerdo con la pregunta y los objetivos planteados, el propósito de la investigación fue conocer el efecto del manejo de errores en la comprensión de un dominio de conocimiento y en la competencia de resoluciónd de problemas, en estudiantes del colegio Psicopedagógico Angeli. Para cuyos efectos se empleó la teoría de la línea recta como tema de estudio. También se utilizó un grupo control, que recibió clases de forma tradicional.

\section{El objeto de aprendizaje}

El objeto de aprendizaje orienta al desarrollo de la competencia de resolución de problemas, tomando como base el planteamiento de los mismos y el dominio de conocimiento apunta al aprendizaje de la teoría de la línea recta. Consta de tres partes: la presentación, un tutor y resolución de problemas. El tutor utiliza retroalimentación KCR para los ejercicios propuestos y la resolución de problemas utiliza retroalimentación ITF.

En este orden de ideas, el aprendizaje se puede definir como la codificación de conocimientos o habilidades en la memoria a largo plazo, de tal manera que se puedan recuperar recuerdos cuando se necesiten (Cooper, 1998). Esta base de conocimiento está organizado en una red -también de conocimiento- bien estructurada y asociada a otras redes de la misma naturaleza, a las cuales se les llama esquema. En la medida en que una persona posea esquemas más detallados y complejos, demuestra más habilidad en un dominio de conocimiento y en la resolución de problemas. Tomando en consideración estas afirmaciones se puede dar respuesta y explicación a las preguntas que planteó esta investigación.

Al comparar el desempeño de los resultados del pre y postest en el dominio de conocimiento, se encontró que en el grupo B fue significativo. En el grupo A, por el contrario, a pesar de obtener un promedio alto no alcanzó la significancia. Tal vez, las carácterísticas del tutor no permitieron la modificación efectiva de los esquemas de ese grupo de estudiantes. Al mismo tiempo la retroalimentación KCR no produjo, en este caso, los resultados de desempeño esperados. Posiblemente, la competencia interpretativa de los estudiantes no fue suficiente para entender a cabalidad el tema. 
Con respecto a la competencia de resolución de problemas sucedió algo parecido. El grupo B mostró un desempeño significativo, comparando las pruebas pretest - postest. El grupo A no logró el valor del Sig. necesario para considerar su desempeño significativo. La retroalimentación ITF no produjo un efecto suficientemente fuerte para inferir que el nivel de competencia fuera superior. Por otro lado, se observó durante la práctica lo mencionado por Aleven y Koedinger (2000): muchos estudiantes ignoraban la función de ayuda y no leían los mensajes de retroalimentación que les brindaba el software. Adicionalmente, se puede mencionar frente al factor tiempo, lo siguiente: los estudiantes del grupo A solo llegaron al nive 2; al parecer, el estudio independiente requiere más tiempo, porque los estudiantes deben recurrir al procesamiento de la información para adquirir el conocimiento.

La solución del problema se dividió en tres fases: comprensión, definición de variables y modelación matemática. Las dos primeras fases se diseñaron para mejorar el desempeño en la fase modelación matemática, que incluye las dos anteriores. Los resultados mostraron que los estudiantes definían las variables con sus respectivas descripciones, sin conectar su significado al contexto del problema. A la hora de expresar la ecuación, no sabían cuáles de las variables -definidas por ellos previamenteasumían la función de variable independiente o pendiente, ni cuál era el punto de corte o la pendiente. Es decir, no tenían el conocimiento que se había estudiado en las dos fases anteriores.

Finalmente, los problemas se presentaron en forma de historia para ser resueltos por ambos grupos. En virtud de lo cual se puede decir que el objeto de aprendizaje conduce al estudiante a construir la competencia de resolución de problemas (en los temas correspondientes) a través de la realización de actividades de procesamiento de información. Bajo este panorama, es un requisito resolver correctamente el paso actual para poder continuar con el siguiente problema. En el proceso de obtención de principios los estudiantes despliegan actividades de comprensión y utilizan esquemas previos (Cooper, 2003) que, combinados con el proceso de metacognición, conducen a la construcción del conocimiento (Cheng, 2003).

\section{Síntesis}

- El grupo B tuvo un desempeño significativo tanto en el dominio de conocimiento como en la resolución de problemas.

- El grupo A no alcanzó el desempeño significativo, ni el dominio de conocimiento en la resolución de problemas.

- No hubo diferencia significativa entre el desempeño de los dos grupos.

- El grupo A llegó hasta el nivel 2 de complejidad. El grupo B, llegó al nivel 4.

La retroalimentación KCR, utilizada en el tutor, no produjo el desempeño esperado en el dominio de conocimiento.

La retroalimentación ITF y el manejo de errores no produjeron un efecto suficientemente fuerte para lograr un desempeño significativo en la resolución de problemas.

- Hubo error de discriminación de concepto en la resolución de problemas, puesto que se confundían los conceptos variables dependiente, variable independiente, punto de corte y pendiente.

- La estrategia que se basó en el procesamiento de información por parte del estudiante, requirió más tiempo que cuando explicaba el profesor.

- No se debe pensar que los objetos de aprendizaje son inútiles. Todo lo contrario, al utilizarlos se deben tener en cuenta las condiciones de uso, por ejemplo, el tiempo.

- Una de las variables críticas en el uso de los objetos de aprendizaje es el descarte de la retroalimentación, antes de leerla. En virtud de lo anterior se debe pensar en estrategias para lograr que se lea.

\section{Bibliografía}

Bannan, B.; Dabbagh, N. y Murphy, K. (2000). "Learning object systems as constructivist learning environments: related assumptions, 
theories and applications", [en línea]. Recuperado de http://reusability.org/read/chapters/bannan-ritland.doc

Calhoun, B. y Wooten, L. (2003). The impact of learning objects. IT 6750: Current Trends and Issues in Instructional Technology.

Chan, M. E. (2001). "Objetos de aprendizaje: una herramienta para la innovación educativa", [en línea]. Recuperado de www.udgvirtual.udg.mx/ apertura/pdfs/.../RevApertura_Dic2002.pdf

Cooper, G. (2003). "Cognitive load theory as an aid for instructional design". En Australian Journal of Educational Technology, vol. 6, núm. 2, pp. 108-113, [en línea]. Recuperado de http://www.ascilite.org.au/ajet/ajet6/cooper.html

Dempsey, J. V. (1988). “The effects of four methods of immediate corrective feedback on retention, discrimination error, and feedback study time in computer-based instruction" [tesis doctoral de Florida State University]. Dissertation Abstracts International, 49, 1434A.

Jonassen, D. H. (2000). “Toward a design theory of problem solving”. En Educational Technology: Research \& Development, vol. 48, núm. 4, pp. 63-85.

Kulhavy, R. W., y Stock, W. A. (1989). "Feedback in written instruction: The place of response certitude". En Educational Psychology Review, vol. 1, núm. 4, pp. 279-308.

Meyer, L. (1986). "Strategies for correcting students' wrong responses". En Elementary School Journal, núm. 87, pp. 227-241.

Mory, H. M. (2004). "Feedback research revisited". En Handbook of research on educational communications and technology, pp. 745-784, [en línea]. Recuperado de http://www.aect.org/ edtech/29.pdf

Narciss, S.; Körndle, H.; Reimann, G. y Müller, C. (2004). "Feedback-seeking and feedback efficiency in web-based learning - How do they relate to task and learner characteristics?" En Gerjets, P.; Kirschner, P.; Elen, J y Joiner, R.
(Eds.), Instructional design for effective and enjoyable computer-supported learning. Proceedings of the first joint meeting of the EARLI SIGs instructional Design and Learning and Instruction with Computers, pp. 377-388. Tuebingen: Knowledge Media Research Center. Recuperado de http:// www.aect.org/edtech/29.pdf

Noonan, J. V. (1984). "Feedback procedures in computer-assisted instruction: Knowledge-ofresults, knowledge-of-correct-response, process explanations, and second attempts after errors" [tesis doctoral de la Universidad de Illinois].

Otero, L. E. (2009). "Efectos de la retroalimentación sobre la competencia de resolución de problemas". En Vestigium. Rev. Acad. Univ., [número especial], pp. 65-73.

Phye, G. D., y Bender, T. (1989). "Feedback complexity and practice: Response pattern analysis in retention and transfer". En Contemporary Educational Psychology, vol. 14, pp. 97-110.

Rohrer L. y Suen H. K. (April, 1999). "Validating measures of structural knowledge through the multitrait - multimethod matrix". En Pennsylvania State University Paper presentation at the Annual Meeting of the American Educational Research Association, Montreal. Recuperado de http://suen.educ.psu. edu/ hsuen/pubs/concept\%20mtmm.pdf

Shavelson, R. J. (1972). "Some aspects of the correspondence between content structure and cognitive structure in physics instruction". En Journal of Educational Psychology, vol. 63, pp. 225-234.

Skinner, B. F. (1958). “Teaching machines”. En Science, vol. 128, pp. 969-977.

Sweller, J. (1988). “Cognitive load during problema solving: Effectos on learning". En Cognitive Science, vol. 12, pp. 257-285.

Valencia, C. y Jiménez, A. (Eds.). (2009). Objetos de Aprendizaje Prácticas y perspectivas educativas. Cali: Pontificia Universidad Javeriana.

Wiley D, 2000, "The Instructional Use of Learning Objects”, [en línea]. Recuperado de http:// www.reusability.org/read 